\title{
ESR1 mutation causes estrogen resistance and puberty delay in women
}

A mutation in ESR1, the gene encoding estrogen receptor $\alpha$, leads to profound estrogen insensitivity and delayed puberty in women, report researchers in The New England Journal of Medicine.

Mutations in ESR1 are rare and have previously only been reported in one man. Quaynor et al. have identified a mutation in an 18-year old white woman with clinical features of estrogen resistance, including elevated serum estrogen levels, absence of breast development despite oral estrogen treatment, small uterus and enlarged multicystic ovaries.

The investigators first measured serum estradiol by immunoassay. "We repeated the estradiol and estrone analysis by liquid chromatography-mass spectometry to confirm that the estrogens were not spuriously elevated; both were 10 -fold above normal values," explains senior investigator Layman.

Next, the researchers sequenced the ESR1 gene, which revealed a homozygous missense mutation in exon 5 . The resulting amino acid change within the ligand-binding domain was predicted to be deleterious, and in vitro experiments showed markedly reduced activity of the mutated receptor. Samples from the patient's parents were not available; however, single nucleotide polymorphism microarray revealed a region of $11 \%$ homozygosity, suggesting that they were second-degree relatives and heterozygous carriers of the same mutation.

ESR1 mutations are not lethal but confer a pronounced estrogen-resistant state, the authors conclude. "Possibly milder phenotypes exist, for example, a subset of patients with infertility might have ESR1 mutations," says Layman.

Elisabeth Kugelberg

Original article Quaynor, S. D. et al. Delayed puberty and estrogen resistance in a woman with estrogen receptor $a$ variant. N. Engl. J. Med. 369, 164-171 (2013) 